\title{
Production of scopularide A in submerged culture with Scopulariopsis brevicaulis
}

\author{
Anu Tamminen', Annemarie Kramer², Antje Labes² and Marilyn G Wiebe ${ }^{1^{*}}$
}

\begin{abstract}
Background: Marine organisms produce many novel compounds with useful biological activity, but are currently underexploited. Considerable research has been invested in the study of compounds from marine bacteria, and several groups have now recognised that marine fungi also produce an interesting range of compounds. During product discovery, these compounds are often produced only in non-agitated culture conditions, which are unfortunately not well suited for scaling up. A marine isolate of Scopulariopsis brevicaulis, strain LF580, produces the cyclodepsipeptide scopularide A, which has previously only been produced in non-agitated cultivation.

Results: Scopulariopsis brevicaulis LF580 produced scopularide A when grown in batch and fed-batch submerged cultures. Scopularide A was extracted primarily from the biomass, with approximately $7 \%$ being extractable from the culture supernatant. By increasing the biomass density of the cultivations, we were able to increase the volumetric production of the cultures, but it was important to avoid nitrogen limitation. Specific production also increased with increasing biomass density, leading to improvements in volumetric production up to 29-fold, compared with previous, non-agitated cultivations. Cell densities up to $36 \mathrm{~g} \mathrm{~L}^{-1}$ were achieved in 1 to $10 \mathrm{~L}$ bioreactors. Production of scopularide A was optimised in complex medium, but was also possible in a completely defined medium.

Conclusions: Scopularide A production has been transferred from a non-agitated to a stirred tank bioreactor environment with an approximately 6-fold increase in specific and 29-fold increase in volumetric production. Production of scopularide A in stirred tank bioreactors demonstrates that marine fungal compounds can be suitable for scalable production, even with the native production organism.
\end{abstract}

Keywords: Marine fungi, Scopulariopsis brevicaulis, Scopularide A, Stirred tank bioreactor

\section{Background}

Marine organisms produce many novel compounds with various biological activities [1,2]. Until recently, much of the research has focused on compounds from marine invertebrates, bacteria, cyanobacteria and dinoflagelates, but interesting compounds have also been isolated from marine fungi $[3,4]$. During product discovery, these compounds are generally identified as extracts from strains growing in non-agitated conditions, which are convenient for large scale product discovery, but not well suited for scaling up [5-7].

A strain of Scopulariopsis brevicaulis (LF580) which was isolated from a marine sponge was shown to produce an interesting cyclodepsipeptide, scopularide A [8]. Scopularide

\footnotetext{
* Correspondence: marilyn.wiebe@vtt.fi

${ }^{1}$ VIT Technical Research Centre of Finland, P.O. Box 1000, FI-02044 VTT, Finland

Full list of author information is available at the end of the article
}

A consists of five amino acids (glycine, L-phenylalanine, Lalanine, L-valine and D-leucine) and hydroxymethyldecanoic acid. It inhibited the growth of both pancreatic and colon tumor cell lines [8] and may be of interest for further studies. Yu et al. [8] grew S. brevicaulis in complex medium (Yeast Malt Peptone, YMP, with $30 \mathrm{~g} \mathrm{~L}^{-1} \mathrm{NaCl}$ ) containing $10 \mathrm{~g} \mathrm{~L}^{-1}$ glucose and $3 \mathrm{~g} \mathrm{~L}^{-1}$ malt extract as the primary carbon sources, for 14 days to produce $7 \mathrm{mg} \mathrm{L}^{-1}$ scopularide A (approximately $0.02 \mathrm{mg} \mathrm{L}^{-1} \mathrm{~h}^{-1}$ ) in non-agitated cultivation conditions (static flasks). Neither the conditions of production nor the biosynthetic pathway were investigated.

S. brevicaulis (teleomorph Microascus brevicaulis, formerly Penicillium brevicaule) is commonly isolated from soil environments. Soil isolates have been used to volatilize arsenic and to methylate antimony [9], and to produce keratinase for degradation of feathers [10] or methioninase, an enzyme with potential medicinal application [11]. S. brevicaulis has been isolated as an opportunistic human 
pathogen, causing infections of skin or nails. Some strains have been isolated from high salt environments or shown to tolerate high concentrations of salt $[12,13]$. It has not often been reported from marine environments [14], nor have there been reports of scopularide production from other isolates, probably because they have not been assessed.

In this paper we demonstrate that scopularide $\mathrm{A}$ is produced by S. brevicaulis LF580 in stirred tank bioreactors (STRs) and describe the growth and scopularide A production of Scopulariopsis brevicaulis LF580 in STRs from $500 \mathrm{~mL}$ to $10 \mathrm{~L}$ scale.

\section{Results and discussion}

\section{Scopularide A was produced in agitated conditions}

Scopularides were produced in shaken flasks in the YMP medium described by [8], but production was variable: varying from 2 to $25 \mathrm{mg} \mathrm{L}^{-1}$ scopularide A. Shaken flasks contained relatively large volumes of culture (up to 50\%) and were agitated under mild conditions, as a first step in the transition from static to agitated cultivation. Thus shaken flasks would have generally been oxygen limited, with variable hyphal morphology, both of which probably contributed to the high degree of variation observed in scopularide A production. However, the range observed indicated that scopularides could also be produced in STRs.

In STR, $29 \pm 3 \mathrm{mg} \mathrm{L}^{-1}$ scopularide A (mean \pm standard error of the mean (sem); $\sim 2.4 \mathrm{mg}[\mathrm{g} \text { biomass }]^{-1}$ ) were produced after $90 \mathrm{~h}$ cultivation (Table 1 ). Both volumetric and specific scopularide A concentrations were improved, relative to the production in static flasks (Table 1) and production was more consistent than in shaken flasks. Similar results were obtained in bioreactors in both labs (i.e. at two independent locations) with reactors varying in size from 0.5 to $10 \mathrm{~L}$ scale. This improvement in scopularide A production in agitated conditions suggested that adequate air supply was important for scopularide A production, as was confirmed with an oxygen-limited bioreactor (10 mg scopularide $\mathrm{A} \mathrm{L}^{-1}, 0.7 \mathrm{mg}$ [g biomass] ${ }^{-1}$, Table 1) in which scopularide A production was comparable to that observed in the static flasks.

The medium supported a maximum specific growth rate of $0.25 \pm 0.01 \mathrm{~h}^{-1}$ and the production of $11.3 \pm 1 \mathrm{~g}$ biomass $\mathrm{L}^{-1}$ (yield $\sim 0.83 \mathrm{~g}$ biomass [g carbohydrate] ${ }^{-1}$, assuming that malt extract contained $63 \%$ available carbohydrate).

\section{Nitrogen-limitation limited scopularide A production}

Scopularide A was primarily retained in the hyphae, with only $7 \pm 1 \%$ of the total being extractable from the culture supernatant at the end of various cultivations. Scopularide A production might therefore be improved by increasing the amount of biomass produced, provided that sufficient oxygen is available, as in STRs.

When S. brevicaulis LF580 was grown in YMP medium with 20 g glucose $\mathrm{L}^{-1}$ without addition of $\left(\mathrm{NH}_{4}\right)_{2} \mathrm{SO}_{4}$ scopularide A production was reduced to $8.1 \mathrm{mg} \mathrm{L}^{-1}$ (Table 1,

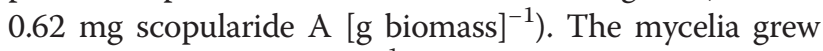
exponentially at $\mu=0.24 \mathrm{~h}^{-1}$ during the first $14 \mathrm{~h}$, but then entered a prolonged deceleration phase, even though more than $14 \mathrm{~g} \mathrm{~L}^{-1}$ glucose still remained. YMP is a weak complex medium which contains approximately $0.76 \mathrm{~g} \mathrm{~L}^{-1}$ amino nitrogen, which is not necessarily all available to an organism, depending on its ability to hydrolyse all peptide bonds. When additional nitrogen $\left(0.75 \mathrm{~g}\left(\mathrm{NH}_{4}\right)_{2} \mathrm{SO}_{4} \mathrm{~L}^{-1}\right)$ was added to the culture at $47 \mathrm{~h}, \mathrm{CO}_{2}$ production immediately increased and the remaining glucose was rapidly

Table 1 Biomass and maximal scopularide A production by S. brevicaulis LF580 in bioreactor cultivations

\begin{tabular}{|c|c|c|c|c|c|}
\hline Condition & Glucose $\left(\mathrm{g} \mathrm{L}^{-1}\right)$ & $\mathrm{C} / \mathrm{N}\left(\mathrm{g} \mathrm{g}^{-1}\right)$ & Biomass $\left(\mathrm{g} \mathrm{L}^{-1}\right)$ & Scopularide $A\left(\mathrm{mg} \mathrm{L}^{-1}\right)^{\dagger}$ & Time (h) \\
\hline Static flask - YMP [8] & 10 & 10 & & 7 & $\sim 336$ \\
\hline $\mathrm{O}_{2}$-limited batch-YMP ${ }^{\dagger \dagger}$ & 11 & 10 & 11 & 10 & 90 \\
\hline Batch - YMP & 11 & 10 & $11 \pm 1$ & $29 \pm 3$ & 90 \\
\hline $\mathrm{N}$-limited batch-YMP & 21 & 15 & $12 \pm 1$ & 8 & 74 \\
\hline Batch - YMP & 20 & 11 & $15 \pm 2$ & $58 \pm 4$ & 90 \\
\hline Batch or fed-batch-YMP & 44 & 10 & $25 \pm 1$ & $142 \pm 20$ & 167 \\
\hline Batch or fed-batch-YMP & 61 & 10 & $33 \pm 1$ & $202 \pm 58$ & 165 \\
\hline Batch - CSS & 17 & 7 & 12 & 44 & 90 \\
\hline Batch - defined, glucose & 17 & 9 & 12 & 41 & 87 \\
\hline Batch - defined, glucose & 40 & 9 & $24 \pm 6$ & $44 \pm 7$ & 100 \\
\hline Batch - defined, xylose & 40 & 9 & $25 \pm 1$ & $54 \pm 2$ & 100 \\
\hline
\end{tabular}

S. brevicaulis LF580 was grown in YMP, CSS or defined medium (and $30 \mathrm{~g}$ Sea Salt $\mathrm{L}^{-1}$ ) in batch or fed-batch culture at $28^{\circ} \mathrm{C}$. Scopularide $\mathrm{A}$ was extracted from the mycelia at the times indicated. C/N values are estimated, as indicated in the methods. Average values are given, with \pm sem for scopularide $A$ and biomass, for cultivations which were carried out in replicate $(n=2$ to 5$)$. Published data from static flasks [8] is provided for comparison.

${ }^{\dagger}$ Determined from total dry biomass, volume of culture remaining and specific scopularide A content of the biomass (see Figure 1).

${ }^{+\dagger} \mathrm{O}_{2}$-limited culture agitated at $300 \mathrm{rpm}$, with $0.6 \mathrm{vvm}$ air. 
consumed, indicating that the culture had been nitrogen limited. Nitrogen limitation would be expected to reduce scopularide production, since nitrogen needed for biomass production would no longer be available for synthesis of non-essential metabolites and nitrogen incorporated into scopularides could be recycled for more important cellular processes. Addition of $1 \mathrm{~g} \mathrm{~L}^{-1}\left(\mathrm{NH}_{4}\right)_{2} \mathrm{SO}_{4}(0.21 \mathrm{~g} \mathrm{~N})$ to YMP with $20 \mathrm{~g} \mathrm{~L}^{-1}$ glucose was sufficient to improve scopularide A production $\left(58 \mathrm{mg} \mathrm{L}^{-1}, 3.6 \mathrm{mg}\right.$ [g biomass $^{-1}$ ). We concluded that the carbon nitrogen ratio $\left(\mathrm{C} / \mathrm{N}, \mathrm{g} \mathrm{g}^{-1}\right)$ should be $\leq 11$ for good scopularide A production.

\section{Production of scopularide $A$ in complex and defined medium with high biomass concentrations}

$S$. brevicaulis LF580 was grown in batch and/or fed-batch cultures with 20-60 g glucose $\mathrm{L}^{-1}$ in YMP medium supplemented with $\left(\mathrm{NH}_{4}\right)_{2} \mathrm{SO}_{4}$. Fed-batch cultures were used to avoid oxygen limitation and glucose repression at high glucose concentrations, before it became apparent that scopularide A was being produced during growth, even in the presence of glucose (Figure 1). Oxygen limitation did not occur in any culture, since agitation speed was automatically adjusted to maintain dissolved oxygen at levels $\geq 30 \%$. Specific scopularide A production was similar in batch and fed-batch cultivations, as was the final concentration of scopularide A (Figure 1) and amount

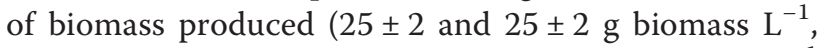
for batch and fed-batch cultures supplied with $44 \mathrm{~g} \mathrm{~L}^{-1}$ glucose, respectively, and $31 \pm 1$ and $36 \mathrm{~g}$ biomass $\mathrm{L}^{-1}$, when provided $61 \mathrm{~g} \mathrm{~L}^{-1}$ glucose).

The supply of glucose in fed-batch cultures did limit biomass production in the $\sim 44 \mathrm{~g} \mathrm{~L}^{-1}$ glucose cultures (Figure 1), resulting in generally higher biomass and scopularide A concentrations in batch than in fed-batch cultures between 40 and $140 \mathrm{~h}$. Since some scopularide A was also produced during the stationary phase of batch cultures, volumetric production rates were similar in both (e.g. $1.1 \pm 0.3$ and $1.0 \pm 0.1 \mathrm{mg} \mathrm{L}^{-1} \mathrm{~h}^{-1}$, in batch and fed-batch cultures with $\sim 44 \mathrm{~g} \mathrm{~L}^{-1}$ glucose, respectively). The slow initial biomass production in fed-batch cultures could be improved with a more optimal feeding profile, but no optimisation was undertaken, since fed-batch did not provide clear benefits. Since results were similar in batch and fed-batch cultures, final production data from the two methods have been combined (Table 1, Figure 2).

Both biomass and scopularide A production increased with increasing glucose provision (Table 1). Up to $202 \pm$ $58 \mathrm{mg} \mathrm{L}^{-1}$ scopularide A were produced in cultures with $61 \mathrm{~g} \mathrm{~L}^{-1}$ glucose (Table 1), which was 29-fold higher than produced in the static flask cultures of $\mathrm{Yu}$ et al. [8]. The
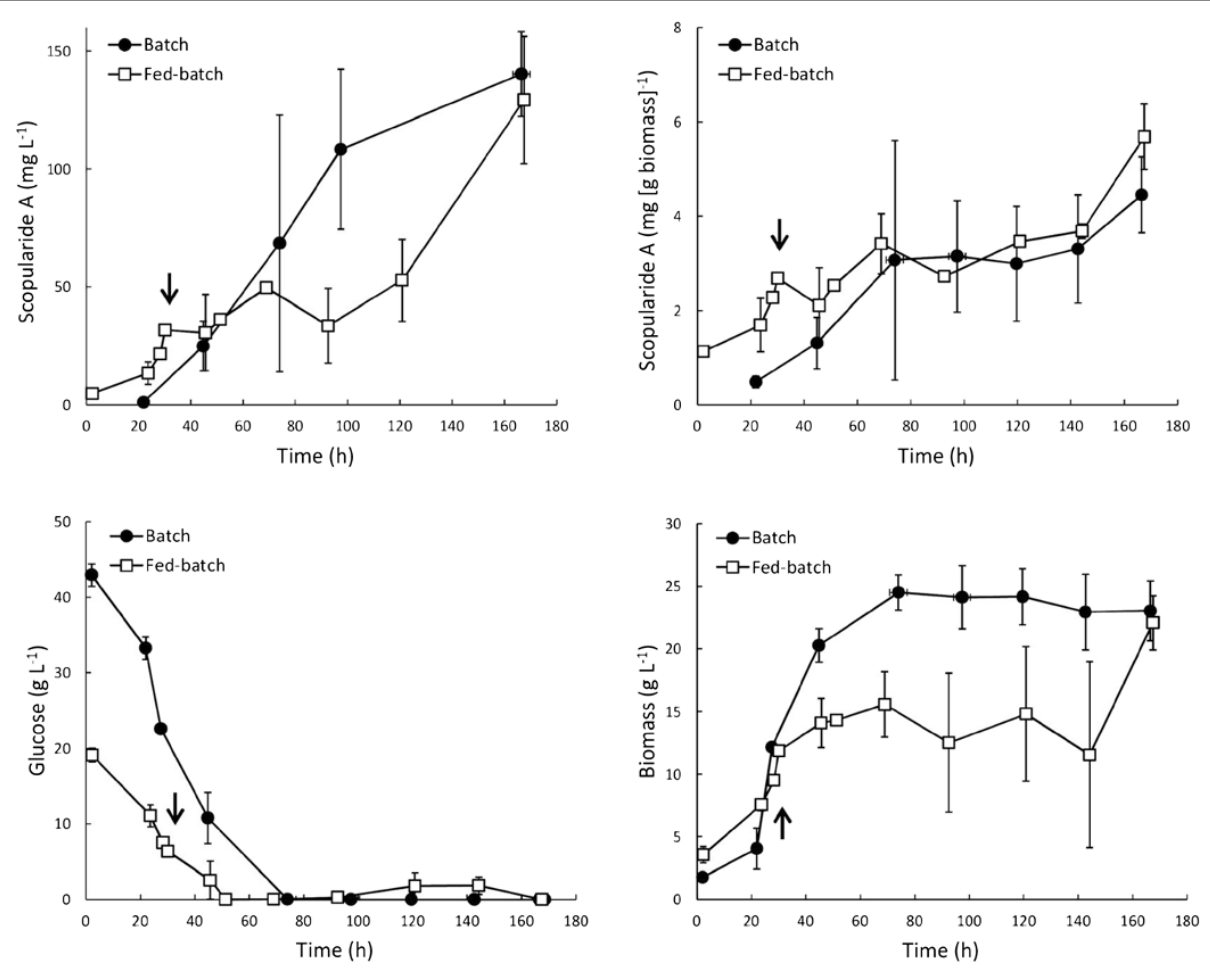

Figure 1 Comparison of batch (solid circle) and fed-batch (open square) cultures of S. brevicaulis LF580. Cultures were provided a final total of $\sim 44 \mathrm{~g} \mathrm{~L}^{-1}$ glucose and were grown in YMP medium supplemented with $\left(\mathrm{NH}_{4}\right)_{2} \mathrm{SO}_{4}$ (Table 1) and $30 \mathrm{~g} \mathrm{~L}^{-1}$ marine salt, as described in the methods. Cultures were agitated at 400-600 rpm to maintain DOT $\geq 30 \%$. Arrows indicate the time at which medium started to be fed to the fed-batch cultures. Error bars represent \pm sem for $n=3$ (batch) and $n=2$ (fed-batch) cultures. 


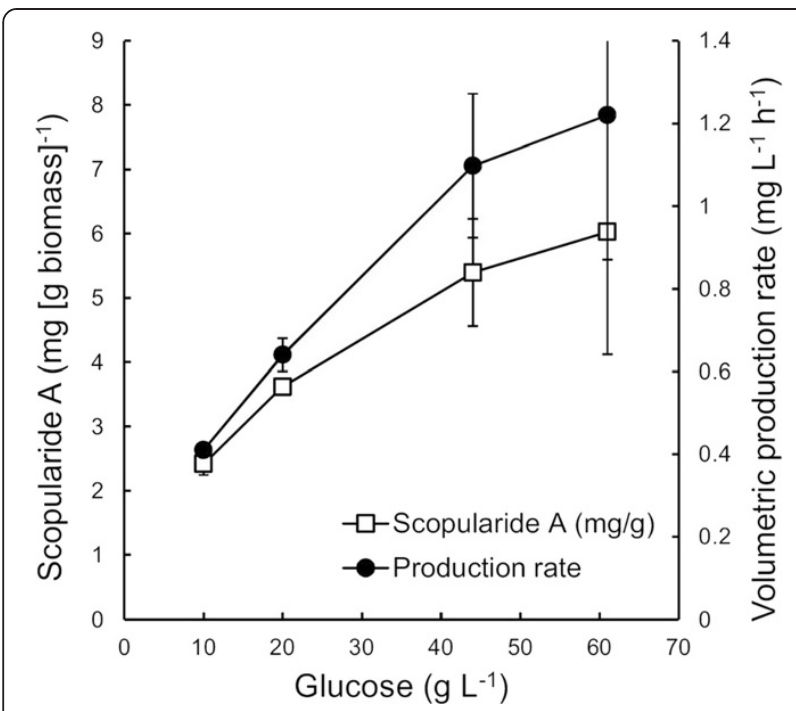

Figure 2 Effect of glucose concentration on specific scopularide A production and the volumetric production rate. Specific scopularide A production and volumetric production rate at time of maximal production (see Table 1). S. brevicaulis LF580 was grown in

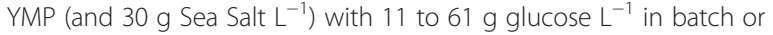
fed-batch culture at $28^{\circ} \mathrm{C}$. Scopularide A was extracted from the mycelia. Error bars are \pm sem for $n=4,2,5$ or 3 independent cultivations with $\sim 10,20,40$ or $60 \mathrm{~g}$ glucose $L^{-1}$, respectively. Where not visible, error bars were smaller than the symbol. volumetric production rate was increased from $0.4 \mathrm{mg} \mathrm{L}^{-1}$ $\mathrm{h}^{-1}$ with $11 \mathrm{~g} \mathrm{~L}^{-1}$ glucose to $1.2 \pm 0.3 \mathrm{mg} \mathrm{L}^{-1} \mathrm{~h}^{-1}$ with $61 \mathrm{~g} \mathrm{~L}^{-1}$ glucose (Figure 2).

Surprisingly, specific scopularide A production (mg $[\mathrm{g} \text { biomass }]^{-1}$ ) also increased with increasing glucose provision (Figure 2). Specific scopularide A production increased from $2.4 \mathrm{mg} \mathrm{g}^{-1}$ with $10 \mathrm{~g}$ glucose $\mathrm{L}^{-1}$ to $6.0 \pm$ $1.9 \mathrm{mg} \mathrm{g}^{-1}$ with $60 \mathrm{~g}$ glucose $\mathrm{L}^{-1}$. Thus, even though there was greater variation in the amount of scopularide A produced in replicate cultures at high biomass concentrations than at low, volumetric production was improved more than expected by the 3 -fold increase in biomass production.

Maximum specific growth rate $\left(0.25 \pm 0.01 \mathrm{~h}^{-1}\right)$ was not affected by increasing the glucose concentration, but the yield of biomass on glucose and malt extract decreased, since yeast extract and soy peptone provided carbon as well as nitrogen for growth, but were added at a constant 3 and $5 \mathrm{~g} \mathrm{~L}^{-1}$, respectively, rather than in proportion to the glucose concentration, with additional nitrogen supplied as $\left(\mathrm{NH}_{4}\right)_{2} \mathrm{SO}_{4}$.

Scopularide A was also produced in chemically defined medium, but at lower concentrations than in the corresponding cultures provided complex medium (Table 1). The specific growth rate of $S$. brevicaulis LF580 was reduced to $0.17 \pm 0.03 \mathrm{~h}^{-1}$ in defined medium with glucose as carbon source, and $0.14 \mathrm{~h}^{-1}$ with xylose as carbon source, whereas biomass production (Table 1) and yield of biomass on carbohydrate were similar. S. brevicaulis LF580 did not grow on lactose (data not shown). The high biomass ( $40 \mathrm{~g} \mathrm{~L}^{-1}$ glucose) cultures were harvested after $100 \mathrm{~h}$ cultivation, after which time only small increases in scopularide A production had occurred in complex medium. This may have been too early for the slower growing defined medium cultures. However, most of the scopularide A in these cultures $\left(47-50 \mathrm{mg} \mathrm{L}^{-1}\right)$ was actually produced within the first $48 \mathrm{~h}$ and therefore we did not expect further production.

Similar amounts of scopularide A were produced on glucose or xylose, a less repressive carbon source, in defined medium (Table 1, 2.3 $\mathrm{mg}$ [g biomass] $^{-1}$ ). Scopularide A was produced in the presence of glucose concentrations up to $51 \mathrm{~g} \mathrm{~L}^{-1}$ in complex medium and its production did not appear to be repressed by glucose. Scopularide A may be considered a secondary metabolite in that it is not part of central metabolic pathways. However, unlike many secondary metabolites [15], scopularide A was produced during growth (Figure 1). Indeed, other peptide-derived fungal metabolites such as penicillin and cephalosporin are also produced during growth when carbon sources other than glucose are supplied [16], or when glucose supply is limited.

\section{Alternative nitrogen sources for scopularide A production}

Yeast extract and soy peptone are relatively expensive sources of nitrogen, but could be replaced with corn steep solids (CSS). Similar amounts of scopularide A (3.6 mg [g biomass] ${ }^{-1}$ with $17 \mathrm{~g} \mathrm{~L}^{-1}$ glucose) were produced with CSS as the N-source, as in YMP. Each of these substrates provides short peptides and amino acids which generally support better growth than inorganic nitrogen sources by reducing the need to synthesise amino acids de novo. Trace elements, vitamins and some lipids are also provided. However, as sources of amino acids, they may also provide precursors (i.e. phyenylalanine, alanine, leucine, valine and glycine) for the scopularides. Yeast extract and soy peptone contain relatively higher amounts of all precursor amino acids than CSS, with the exception of alanine. The similar production of scopularides in CSS as in YMP suggests that amino acids from these sources were not serving as direct precursors for scopularide A production. More biomass was produced in YMP $\left(15 \pm 2 \mathrm{~g} \mathrm{~L}^{-1}\right)$ than in CSS $\left(12 \mathrm{~g} \mathrm{~L}^{-1}\right)$, and thus volumetric scopularide A production was higher in YMP than in CSS (Table 1), which may indicate that the nitrogen in CSS was less accessible for growth than the peptides in yeast extract and soy peptone. None-the-less, Scopularide A can be produced in medium in which nitrogen is provided as amino acids from various complex sources or as ammonium, as in defined medium. 


\section{Conclusions}

Scopularide A production has been transferred from a non-agitated to a stirred tank bioreactor environment with approximately 6-fold increase in specific and 29-fold increase in volumetric production. Production of scopularide A in STRs demonstrates that marine fungal compounds can be suitable for scalable production, even with the native production organism, and similar strategies should be applied to enhance production of other recently discovered cyclic peptides which have only been produced in flask culture (e.g. [17-20]).

\section{Methods}

\section{Strain}

Scopulariopsis brevicaulis LF580 was obtained from the culture collection of the Kiel Center for marine natural products at GEOMAR, Helmholtz Centre for Ocean Research Kiel. Stock cultures were maintained as conidia suspended in $20 \% \mathrm{v} / \mathrm{v}$ glycerol, $0.8 \% \mathrm{w} / \mathrm{v} \mathrm{NaCl}$ with $\sim 0.025 \% \mathrm{v} / \mathrm{v}$ Tween 20 at $-80^{\circ} \mathrm{C}$. Spores were generated from cultures growing on agar-solidified YMP medium containing $30 \mathrm{~g} \mathrm{~L}^{-1}$ Sea Salt (Tropic Marin ${ }^{\circ}$, Germany).

\section{Media}

Yeast Malt Peptone (YMP, containing 3 g yeast extract $\mathrm{L}^{-1}$, $3 \mathrm{~g}$ malt extract $\mathrm{L}^{-1}$ and $5 \mathrm{~g}$ soy peptone $\mathrm{L}^{-1}$ ) medium, modified from a yeast medium described by Wickerham [21] was used as the basic growth medium for S. brevicaulis. Glucose $\left(10-66 \mathrm{~g} \mathrm{~L}^{-1}\right)$ was provided as the primary carbon source. For agar solidified medium, YMP contained $10 \mathrm{~g}$ glucose $\mathrm{L}^{-1}$ and $15 \mathrm{~g}$ agar $\mathrm{L}^{-1}$. Yeast extract and soy peptone were replaced with corn steep solids (CSS, $8 \mathrm{~g} \mathrm{~L}^{-1}$ ) to assess the effect of an alternative peptide source. Media for high cell density cultures were supplemented with $\left(\mathrm{NH}_{4}\right)_{2} \mathrm{SO}_{4}\left(1\right.$ to $\left.9 \mathrm{~g} \mathrm{~L}^{-1}\right)$ in proportion to the additional glucose provided, to ensure that cultures would not become N-limited.

The defined medium of Vogel [22], with $3.3 \mathrm{~g} \mathrm{~L}^{-1}$ $\left(\mathrm{NH}_{4}\right)_{2} \mathrm{SO}_{4}$ substituted for $\mathrm{NH}_{4} \mathrm{NO}_{3}$ and glucose, xylose or lactose as the carbon source, was used to assess growth and scopularide production in defined medium. Supplementation of defined medium with CSS $\left(5 \mathrm{~g} \mathrm{~L}^{-1}\right)$ was also tested.

The composition of feed for fed-batch cultures is described in Table 2.

Media for pre-cultures contained $4 \mathrm{~g}$ agar $\mathrm{L}^{-1}$ to facilitate filamentous growth. All media also contained $30 \mathrm{~g}$ Tropic Marin ${ }^{\circ}$ Sea Salt $\mathrm{L}^{-1}$.

For determination of approximate $\mathrm{C} / \mathrm{N}$ ratios, malt extract was assumed to contain $63 \%$ carbohydrate [23] and $1.1 \%$ total $\mathrm{N}$ [24], yeast extract to contain $9.8 \%$ total $\mathrm{N}$ [24], soy peptone $8.7 \%$ total $\mathrm{N}$ [24] and CSS $7.7 \%$ total $\mathrm{N}$ (based on information from Cerestar). Peptides (amino acids) were assumed to contain 47\%
Table 2 Composition of the feed and the feeding rates used for fed-batch cultures

\begin{tabular}{lccccc}
\hline Reactor & \multicolumn{3}{c}{ Feed composition } & & \\
\cline { 2 - 5 } & $\begin{array}{c}\text { Glucose } \\
\left(\mathbf{g ~ L}^{-1}\right)\end{array}$ & $\begin{array}{c}\left(\mathrm{NH}_{\mathbf{4}}\right)_{\mathbf{2}} \mathbf{S O}_{\mathbf{4}} \\
\left(\mathbf{g ~ L}^{-1}\right)\end{array}$ & $\begin{array}{c}\text { Sea salt* } \\
\left(\mathbf{g ~ L}^{-1}\right)\end{array}$ & $\begin{array}{c}\text { Batch } \\
\text { volume }(\mathbf{L})\end{array}$ & $\begin{array}{c}\text { Feed rate } \\
\left(\mathbf{m L ~ h ~}^{-1}\right)\end{array}$ \\
\hline Qplus & 100 & 21 & 30 & 0.75 & 2.4 \\
CT5-2 & 220 & 50 & 30 & 4.5 & 5.5 \\
C10-3 & 260 & 39 & 30 & 5 & 4.2 \\
\hline
\end{tabular}

S. brevicaulis LF580 was grown in fed-batch cultures in 1 (Qplus), 5 (CT5-2) and 10 (C10-3) L bioreactors.

${ }^{*}$ Tropic Marin ${ }^{\oplus}$.

carbon and CSS to also contain 5\% carbohydrate (based on information from Cerestar).

\section{Cultural conditions}

To assess scopularide production in shaken flasks, flasks (20 to $2000 \mathrm{~mL}$, containing 10 to $1000 \mathrm{~mL}$ medium) were inoculated with conidial suspensions and incubated at $28^{\circ} \mathrm{C}, 120 \mathrm{rpm}$, for up to $170 \mathrm{~h}$.

Pre-cultures for bioreactors were grown in 250 to $1000 \mathrm{~mL}$ Erlenmeyer flasks containing 20\% medium on a volume basis, at $30^{\circ} \mathrm{C}, 200 \mathrm{rpm}$. Flasks were inoculated with conidial suspensions to give final concentrations of $3.4 \times 10^{5}$ conidia $\mathrm{mL}^{-1}$. Pre-cultures were allowed to grow for $48 \mathrm{~h}\left(\sim 19 \mathrm{~g}\right.$ biomass $\left.\mathrm{L}^{-1}\right)$. Fermenters were inoculated with $10 \%$ final volume.

S. brevicaulis was grown in $500 \mathrm{~mL}$ (Multifors, max working volume $500 \mathrm{~mL}$, Infors $\mathrm{HT}$, Switzerland), $1 \mathrm{~L}$ (Biostat Qplus, max working volume 1.0 L, Sartorius AG, Germany), 5 L (Sartorius CT5-2, max working volume $5 \mathrm{~L}$, Germany) and $10 \mathrm{~L}$ (Sartorius Biostat C10-3, max working volumes $10 \mathrm{~L}$, Germany) bioreactors. Bioreactors were maintained at $28^{\circ} \mathrm{C}$, with $700-800$ (Multifors), 500 to 1200 (Biostat Qplus), 400 to 600 (CT5-2), or 400 to 800 (Biostat C10-3) rpm agitation and 0.5 (1-10 L cultures) or 1.7 (0.5 L cultures) volume gas (volume culture $)^{-1} \mathrm{~min}^{-1}$ (vvm). Agitation in 1 to $10 \mathrm{~L}$ bioreactors was adjusted automatically to maintain the dissolved oxygen tension at or above $30 \%$. The oxygen-limited culture was carried out in the Multifors bioreactor with $300 \mathrm{rpm}$ agitation and 0.6 vvm aeration. Culture $\mathrm{pH}$ was kept constant at $\mathrm{pH} 7.0$ by the addition of sterile $1 \mathrm{M} \mathrm{KOH}$ or $1 \mathrm{M} \mathrm{H}_{3} \mathrm{PO}_{4}$. Polypropylene glycol (1:1 mixture of $M_{n} \sim 1000$ and $M_{n} \sim 2000$, [25]) was added to control foam production.

Fed-batch cultures which were fed to a final concentration of $\sim 44 \mathrm{~g}$ glucose $\mathrm{L}^{-1}$ were inoculated as batch cultures with $20 \mathrm{~g} \mathrm{~L}^{-1}$ glucose. Feeding was initiated at approximately $30 \mathrm{~h}$, when glucose concentrations were $<9 \mathrm{~g} \mathrm{~L}^{-1}$. Fed-batch cultures which were fed to a final concentration of $\sim 61 \mathrm{~g}$ glucose $\mathrm{L}^{-1}$ were inoculated as batch cultures with $40 \mathrm{~g} \mathrm{~L}^{-1}$ glucose, since no oxygen-limitation had occurred in the $40 \mathrm{~g} \mathrm{~L}^{-1}$ batch cultures. Feeding was initiated at approximately $47 \mathrm{~h}$. 
Glucose was fed at rates which provided $\sim 0.24 \mathrm{~g} \mathrm{~L}^{-1} \mathrm{~h}^{-1}$. The feed rate and concentration of glucose and ammonium in the feed (Table 2) were determined by the volume of the bioreactor and the capacity of the feed pumps associated with each bioreactor.

Samples were removed at intervals and mycelium was separated from the supernatant by centrifugation (3500 rpm 15 min, Eppendorf AG, centrifuge 5810 R, Germany). Mycelia were washed twice in water by centrifugation and freeze-dried (Christ, Freeze Drier, alpha 1-4 LD plus, Germany) to determine the biomass dry weight. Freeze-dried biomass was used for compound extraction.

Maximum specific growth rates were calculated from $\mathrm{CO}_{2}$ evolution rates. $\mathrm{CO}_{2}$ in the outlet gas was measured continuously in an Omnistar 16 quadrupole mass spectrometer (Balzers AG, Liechtenstein) calibrated with 3\% $\mathrm{CO}_{2}$ in $\mathrm{Ar}$, a Prima Pro Process mass spectrometer (Thermo Scientific, UK) calibrated with $3 \% \mathrm{CO}_{2}$ in $\mathrm{Ar}$, $5 \% \mathrm{CO}_{2}$ with $0.99 \%$ Ar and $15 \% \mathrm{O}_{2}$ in $\mathrm{N}_{2}, 20 \% \mathrm{O}_{2}$ plus $20 \% \mathrm{Ar}$ in $\mathrm{N}_{2}$, and $0.04 \%$ ethanol in $\mathrm{N}_{2}$, or with a photoacoustic IR gas analyser (Innova-1313/LumaSense, United States) with air as reference.

\section{Chemical analyses}

Scopularide A was extracted from mycelia or culture supernatant with ethyl acetate. Freeze-dried mycelia (10-50 mg) were fragmented in the presence of $1.8 \mathrm{~mL}$ ethyl acetate and 2 steel balls in a MM301 ball mill $(2 \times 3$ minutes at 20 $1 / \mathrm{S}$, Retsch $\mathrm{GmbH}$, Germany). Samples were centrifuged $10 \mathrm{~min}$ at $10000 \mathrm{rpm}$ (Eppendorf AG centrifuge 5430) and the ethyl acetate phase transferred to a clean microfuge tube. Alternatively, scopularide A was extracted from $20 \mathrm{~mL}$ culture supernatant, from which mycelia had been removed by centrifugation, by vortexing with $20 \mathrm{~mL}$ ethyl acetate. Phases were separated by centrifugation (3500 rpm $15 \mathrm{~min}$, Eppendorf AG, centrifuge $5810 \mathrm{R}$ ) and the ethyl acetate layer was retained. Ethyl acetate was evaporated under nitrogen and the solids re-dissolved in $200 \mu \mathrm{L}$ HPLC grade methanol.

Ethyl acetate extracts were analysed by UPLC (ACQUITY UPLC, Waters) using a C18 UPLC column $(1.7 \mu \mathrm{m}$, $2.1 \mathrm{~mm} \times 100 \mathrm{~mm}$, Waters; solvent isocratic (A) 70\% $(\mathrm{v} / \mathrm{v})$ acetonitrile, (B) $5 \%(\mathrm{v} / \mathrm{v})$ formic acid in $\left.\mathrm{H}_{2} \mathrm{O}\right)$, and detected with a UV detector.

The concentration of D-glucose in culture supernatant was determined by HPLC using a Fast Acid Analysis Column $(100 \mathrm{~mm} \times 7.8 \mathrm{~mm}$, BioRad Laboratories, Hercules, CA) linked to an Aminex HPX-87H organic acid analysis column (300 $\mathrm{mm} \times 7.8 \mathrm{~mm}, 55^{\circ} \mathrm{C}$, BioRad Laboratories) with $2.5 \mathrm{mM} \mathrm{H}_{2} \mathrm{SO}_{4}$ as eluant and a flow rate of

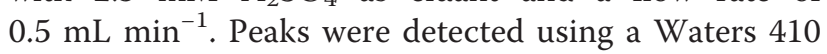
differential refractometer. The concentration of ammonium was measured using a Potentiometer, Lab in the bag $\mathrm{P}$ (C-CIT.AG, Switzerland).

\section{Competing interests}

The authors declare that they have no competing interests.

\section{Authors' contributions}

This study was conceived by MGW and AT. AT carried out cultivations and extractions at VTT. AL and AK carried out cultivations and extractions from flasks and bioreactors with $10 \mathrm{~g} \mathrm{~L}^{-1}$ glucose at GEOMAR. AT and MGW wrote the manuscript. All authors read and approved the final manuscript.

\section{Acknowledgements}

This study was financially supported by the European Union through the $7^{\text {th }}$ Framework KBBE project MARINE FUNGI (project no. 265926). We thank Airi Hyrkäs, Merja Aarnio and Annika Majanen for technical assistance and Atte Mikkelson for discussions concerning the UPLC method.

\section{Author details}

${ }^{1}$ VTT Technical Research Centre of Finland, P.O. Box 1000, FI-02044 VTT, Finland. ${ }^{2}$ Kiel Centre for Marine Natural Products KiWiZ, GEOMAR Helmholtz Centre for Ocean Research, Kiel, Am Kiel-Kanal 44, Kiel 24106, Germany.

Received: 9 March 2014 Accepted: 10 June 2014

Published: 18 June 2014

\section{References}

1. Kobayashi J, Ishibashi M: Bioactive metabolites of symbiotic marine microorganisms. Chem Rev 1993, 93:1753-1769.

2. Proksch P, Edrada RA, Ebel R: Drugs from the seas - current status and microbiological implications. Appl Microbiol Biotechnol 2002, 59:125-143.

3. Bhadury P, Mohammad BT, Wright PC: The current status of natural products from marine fungi and their potential as anti-infective agents. J Ind Microbiol Biotechnol 2006, 33:325-337.

4. Tarman K, Lindequist U, Wende K, Porzel A, Arnold N, Wedssjohann LA: Isolation of a new natural product and cytotoxic and antimicrobial activities of extracts from fungi of Indonesian marine habitats. Mar Drugs 2011, 9:294-306.

5. Le Ker C, Petit K-E, Biard J-F, Fleurence J: Search for hydrophilic marine fungal metabolites: a rational approach for their production and extraction in a bioactivity screening context. Mar Drugs 2011, 9:82-97.

6. Cai M, Zhou X, Lu J, Fan W, Niu C, Zhou J, Sun X, Kang L, Zhang Y: Enhancing aspergiolide $A$ production from a shear-sensitive and easy-foaming marine-derived filamenous fungus Aspergillus glaucus by oxygen carrier addition and impeller combination in a bioreactor. Bioresour Technol 2011 , 102:3584-3586.

7. Bringmann G, Gulder TAM, Lang G, Schmitt S, Stöhr R, Wiese J, Nagel K, Imhoff JF: Large-scale biotechnological production of the antileukemic marine natural product sorbicillactone A. Mar Drugs 2007, 5:23-30.

8. Yu Z, Lang G, Kajahn I, Schmaljohann R, Imhoff JF: Scopularides A and B, cyclodepsipeptides from a marne sponge-derived fungus, Scopulariopsis brevicaulis. J Nat Prod 2008, 71:1052-1054

9. Andrewes P, Cullen WR, Feldmann J, Koch I, Polishchuk E, Reimer KJ: The production of methylated organoantimony compounds by Scopulariopsis brevicaulis. Appl Organometal Chem 1998, 12:827-842.

10. Anbu P, Gopinath SCB, Hilda A, Lakshmipriya T, Annadurai G: Optimization of extracellular keratinase production by poultry farm isolate Scopulariopsis brevicaulis. Bioresour Technol 2007, 98:1298-1303.

11. Khalaf SA, El-Sayed ASA: I-Methioninase production by filamentous fungi: i-screening and optimization under submerged conditions. Curr Microbiol 2009, 58:219-226.

12. Mudau MM, Setati ME: Screening and identification of endomannanaseproducing microfungi from hypersaline environments. Curr Microbiol 2006, 52:477-481.

13. Yoder JA, Benoit JB, Zettler LW: Effects of salt and temperature on the growth rate of a tick-associated fungus, Scopulariopsis brevicaulis bainier (Deuteromycota). Internat J Acarol 2003, 29:265-269.

14. Ding B, Yin Y, Zhang F, Li Z: Recovery and phylogenetic diversity of culturable fungi associated with marine sponges Clathrina luteoculcitella and Holoxea sp. in the South China Sea. Mar Biotechnol 2011, 13:713-721. 
15. Martin JF, Demain AL: Control of antibiotic biosynthesis. Microbiol Rev 1980, 44:230-251.

16. Revilla G, López-Nieto MJ, Luengo JM, Martín JF: Carbon catabolite repression of penicillin biosynthesis by Penicillium chrysogenum. J Antibiot (Tokyo) 1984, 37:781-789.

17. Liu S, Shen Y: A new cyclic peptide from the marine fungal strain Aspergillus sp. AF119. Chem Nat Compd 2011, 47:786-788.

18. Gulder TAM, Hong H, Correa J, Egereva E, Wiese J, Imhoff JF, Gross H: Isolation, structure elucidation and total synthesis of lajollamide A from the marine fungus Asteromyces cruciatus. Mar Drugs 2012, 10:2912-2935.

19. Pérez-Victoria I, Martín J, González-Menéndez V, de Pedro N, El Aouad N, Ortiz-López FJ, Tormo JR, Platas G, Vicente F, Bills GF, Genilloud O, Goetz MA, Reyes F: Isolation and structural elucidation of cyclic tetrapeptides from Onychocola sclerotic. J Nat Prod 2012, 75:1210-1214.

20. Silber J, Ohlendorf B, Labes A, Näther C, Imhoff JF: Calcaripeptides A-C, cyclodepsipeptides from a Calcarisporium strain. J Nat Prod 2013, 76:1461-1467.

21. Wickerham L: Taxonomy of yeasts. US Dept Agric Wash DC Techn Bull 1951, 1029:1-56

22. Vogel HJ: A convenient growth medium for Neurospora (Medium N). Microb Genet Bull 1956, 243:112-119.

23. Neogen malt extract. http://www.neogen.com/Acumedia/pdf/Prodlnfo/ 7341_Pl.pdf.

24. Bridson EY: The Oxoid Manual 8th Edition. Basingstoke: Oxoid Limited; 1998.

25. Wiebe MG, Robson GD, Shuster J, Trinci APJ: Evolution of a recombinant (glucoamylase-producing) strain of Fusarium venenatum A3/5 in chemostat cultures. Biotechnol Bioeng 2001, 73:146-156.

doi:10.1186/1475-2859-13-89

Cite this article as: Tamminen et al: Production of scopularide A in submerged culture with Scopulariopsis brevicaulis. Microbial Cell Factories 2014 13:89.

\section{Submit your next manuscript to BioMed Central and take full advantage of:}

- Convenient online submission

- Thorough peer review

- No space constraints or color figure charges

- Immediate publication on acceptance

- Inclusion in PubMed, CAS, Scopus and Google Scholar

- Research which is freely available for redistribution 